\title{
Postsaturation dynamics and superluminal propagation of a superradiant spike in a free-electron laser amplifier
}

\author{
Xi Yang, ${ }^{1}$ Najmeh Mirian, ${ }^{2}$ and Luca Giannessi ${ }^{2,3}$ \\ ${ }^{1}$ National Synchrotron Light Source II, Brookhaven National Laboratory, Upton, New York 11973, USA \\ ${ }^{2}$ Elettra-Sincrotrone Trieste, Strada Statale 14-km 163,5, 34149 Basovizza, Trieste, Italy \\ ${ }^{3}$ Istituto Nazionale di Fisica Nucleare, Laboratori Nazionali di Frascati, \\ Via Enrico Fermi 45, 00044 Frascati, Italy
}

(Received 3 October 2019; published 13 January 2020)

\begin{abstract}
We study the dynamics of an isolated spike of radiation in superradiant regime. We show that conditions exist where the pulse moves with a group velocity larger than the velocity of light in vacuum and is followed by a pedestal resulting from a complex postsaturation dynamics. The tail is constituted by a train of subpulses with both transverse and longitudinal coherence and decaying amplitudes. We analyze the dynamical conditions leading to the formation of the main pulse and the tail. We study the correlation of the tail structure with the longitudinal phase space of the electrons, and provide a recipe to partially suppress this tail.
\end{abstract}

DOI: 10.1103/PhysRevAccelBeams.23.010703

\section{INTRODUCTION}

Free-electron lasers (FELs) delivering brilliant VUV-Xray pulses with femtosecond temporal resolution are providing unique opportunities in the investigation of fast processes in physics, chemistry, and biology [1-6]. These FELs typically operate in single pass, high gain regime; the electrons, interacting with the self-emitted radiation in a long undulator, are periodically modulated at the resonant wavelength and amplify exponentially the radiation with power folding length $L_{g}$ scaling inversely with the Pierce parameter $\rho_{\text {fel }}$ [7]. The power extracted at saturation is $P_{s} \sim 1.6 \rho_{\mathrm{fel}} P_{e}$, where $P_{e}=\gamma m_{0} c^{2} I_{p} / e_{0}$ is the power carried by the electron beam $\left(e_{0}\right.$ and $m_{0}$ are electron charge and rest mass respectively, $I_{p}$ is the peak current and $\gamma=E_{b} / m_{0} c^{2}$ is the relativistic factor of a beam of average energy $E_{b}$ ) [8]. The difference in the propagation velocities of the electrons and of the radiation (slippage) limits the gain bandwidth (GB) of the process. This imposes a lower limit to the pulse duration $\sigma_{t} \simeq l_{c} / c$, where $l_{c}=$ $\lambda_{0} /\left(4 \pi \rho_{\text {fel }}\right)$ is known as cooperation length [9], where $\lambda_{0}=$ $\lambda_{u}\left(1+K^{2}\right) / 2 \gamma^{2}$ is the resonant wavelength, and where $\lambda_{u}$ and $K$ are the undulator period and root mean square (r.m.s.) strength respectively. Many scientific opportunities become accessible when the pulse duration is at the femtosecond level or even shorter, as resolving the ultrafast

Published by the American Physical Society under the terms of the Creative Commons Attribution 4.0 International license. Further distribution of this work must maintain attribution to the author(s) and the published article's title, journal citation, and DOI. electron dynamics in charge-transfer processes in molecules [10] or resolving the structure of proteins that cannot be crystallized [11]; some of these applications need a short pulse combined with a challenging large number of photons $[12,13]$. We may roughly estimate the number of photons in a cooperation length at saturation as $n_{\mathrm{ph}} \approx \sqrt{2 \pi} \sigma_{t} P_{s} / \hbar \omega_{0} \approx 27 \gamma I_{p}[\mathrm{~A}]\left(\lambda_{0}[\AA]\right)^{2}$. At $1 \AA$, with a beam energy of $17 \mathrm{GeV}$ and a peak current $I_{p}=4 \mathrm{kA}$ (roughly the peak values for LCLS and XFEL), we have $n_{p h} \approx 3.6 \times 10^{9}$, i.e., about two orders of magnitude lower than the requirement for time resolved $x$-ray diffraction imaging of isolated molecules [12]. Several schemes to obtain femtosecond or sub-fs pulses were proposed and in some cases demonstrated [14-21]. Regardless of the generation method, the challenge of increasing the FEL peak power of an isolated spike beyond the saturation limit $P_{s}$ requires a full understanding of saturation and post saturation dynamics. The post-saturation dynamics of a superradiant spike was first studied in $[22,23]$. The field shows the features of a self-similar propagating wave, with peak power exceeding $P_{s}$ and duration shorter than $l_{c} / c$. When the spike reaches saturation, the propagation velocity increases [24], the spectrum of the radiation broadens and the time-bandwidth product grows for the formation of a pedestal with a complicated pattern in the tail of the pulse $[25,26]$. In this paper, we investigate the dispersive properties of the modulated electron beam, showing that the pulse moves with a group velocity larger than the speed of light in vacuum, similar to other fast-light phenomena observed in media with anomalous dispersion [27]. We investigate the formation of the pulse tail structure and its correlation to the longitudinal phase space of the beam, providing a recipe to 
reduce the energy carried by the tail. This may have important practical implications, allowing a substantial reduction of the total pulse duration with a time-bandwidth product closer to the Fourier limit.

\section{FEL DYNAMICAL EQUATIONS}

The system is described by the coupled MaxwellLorentz equations in the slowly varying envelope approximation $[23,28]$.

$$
\begin{aligned}
& \frac{\partial \theta_{j}}{\partial u}=\nu_{j}, \quad \frac{\partial \nu_{j}}{\partial u}=-\left[a(\zeta, u) e^{i \theta_{j}}+\text { c.c. }\right], \\
& \left(\frac{\partial}{\partial u}+\frac{\partial}{\partial \zeta}\right) a(\zeta, u)=-b(\zeta, u) .
\end{aligned}
$$

The electric field $E(z, t)$ is expressed in terms of a slowly varying variable $a(z, t)=E(z, t) e^{-i\left(k_{r} z-\omega_{r} t\right)}$. The FEL power $P_{\text {fel }}$ is linked to $a$ by the relation $P_{\text {fel }}=|a|^{2} \rho_{\text {fel }} P_{e}$. The electron dynamics is coupled to the optical field $a$ via the bunching factor $b(\zeta, u)=\left.\left\langle e^{-i \theta_{j}}\right\rangle_{n_{e}}\right|_{\zeta, u}$, being $n_{e}$ the number of particles at the position $(\zeta, u)$. Each electron has ponderomotive phase and energy $\left(\theta_{j}=\left[\left(k_{u}+k_{r}\right)\left(l_{2} \zeta+l_{1} u\right)-\right.\right.$ $\left.\left.k_{r} l_{1} u / \beta_{z}\right], \nu_{j}=\frac{d \theta_{j}(u)}{d u}\right)$, where the coordinates $u=\beta_{z} c t / l_{1}$ and $\zeta=\left(z-\beta_{z} c t\right) / l_{2} \quad$ (with $l_{1,2}=\lambda_{u, 0} /\left(4 \pi \rho_{\mathrm{fel}}\right)$ and $\left.k_{u, r, 0}=2 \pi / \lambda_{u, r, 0}\right)$, are the normalized beam position along the undulator and the relative particle position in a frame drifting with the average longitudinal velocity of the electron bunch $c \beta_{z}=c\left(1-\left(1+K^{2}\right) / 2 \gamma^{2}\right)$. These equations can be solved perturbatively in small field regime [7,29,30], strong field solutions were investigated including higher order components of the field [31], with the Green function method in [32], or by reduction of the nonlinear, partial differential equations to a set of self-similar equations in [33], but none of these methods is capable of reproducing the entire details of the solution in deep saturation. We directly integrate Eq. (1) using a numerical approach relying on the code PERSEO [34]. We assume an ideal, uniformly distributed current with a null beam energy spread. The system of Eq. (1) is expressed in dimensionless units and depends only on the initial field $a(\zeta, 0)$ and bunching factor $b(\zeta, 0)$. We trigger the formation of the isolated spike by setting the initial condition $a(\zeta, 0)=$ $a_{0} \exp \left[-\left(\zeta-\zeta_{0}\right)^{2} / 4 \sigma_{\zeta 0}^{2}\right]$ and we impose a "quiet start" to the particles distribution, i.e., $b(\zeta, 0)=0$ and $\partial b(\zeta, 0) / \partial u=0$; this is equivalent to an FEL amplifier seeded by $a(\zeta, 0)$. A similar result would be reached by starting from $a(\zeta, 0)=0$ and by setting a non-null initial bunching factor $b(\zeta, 0)$. The condition $\sigma_{\zeta 0}=1$ corresponds to an initial r.m.s. pulse duration equal to $l_{c} / c$, i.e., the minimum pulse duration supported by the bandwidth of the amplification from shotnoise to saturation in exponential gain regime. The cases characterized by the condition $\sigma_{\zeta 0}<1$ share a similar postsaturation dynamics, as the exponential growth will
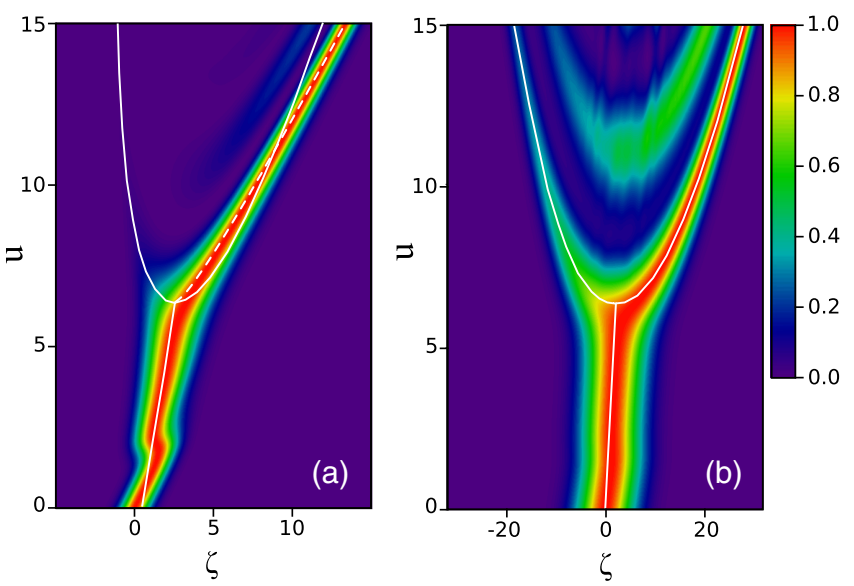

FIG. 1. Radiation power vs $\zeta, u$ for two different initial conditions, (a): $\sigma_{\zeta 0}=0.82$, (b): $\sigma_{\zeta 0}=5$. The continuous white line represents Eq. (1), where the $u_{s}$ position was set to match the saturation splitting position. The white line represents the peak position, drifting with velocity $v_{e}=1 / 3$ before saturation, and splitting for $u>u_{s}$ into the two roots of Eq. (4). The white dashed line for $u>u_{s}$ is the function $\zeta_{+c}(u)$. See text for more details.

produce a similar condition at the onset of saturation with $\sigma_{\zeta 0} \approx 1$.

In Fig. 1, the longitudinal profile of the radiation power vs the electron-beam coordinate $\zeta$ as it propagates along $u$ is shown in two situations: starting from $\sigma_{\zeta 0}=0.82$ in panel (a) and $\sigma_{\zeta 0}=5$ in panel (b). The normalized initial field amplitude is $a_{0}=0.031$ in both panels, and the intensity is normalized at each step in $u$.

At small $u$, the field first induces an energy modulation on the beam, then converted into density modulation by the undulator dispersion. This density modulation is the source term $b(\zeta, u)$ in the second of Eq. (1). The limited gain bandwidth causes a growth of the pulse duration $\sigma_{\zeta}(u) \sim$ $\left(\sigma_{\zeta 0}^{2}+\sqrt{3} u / 18\right)^{\frac{1}{2}}[35] ;$ at saturation $\left(u=u_{s} \approx 6\right)$ the duration is $\sigma_{\zeta s}=\sigma_{\zeta}\left(u_{s}\right)$ and the pulse splits into two modes, the front one propagates over fresh electrons, the rear one interacts with the modulated beam carrying a larger energy spread. The behavior of this second mode depends on the ratio between the gain bandwidth and the pulse bandwidth, i.e., on $\sigma_{\zeta 0} \gtreqless 1$. The regime $\sigma_{\zeta 0}>1$, in Fig. 1(b), was studied in [36] and was later proposed in a seeded FEL in combination with a chirped seed, to generate multiple pulses separated in frequency [37,38]. When $\sigma_{\zeta 0}<1$, the amplitude of the rear mode is reduced and the front mode duration is determined by the slippage distance covered by the light in a quarter of the synchrotron period. At the splitting, this condition corresponds to $\sigma_{\zeta} \approx \sigma_{\zeta s} / 2$. The following evolution is characterized by a pulse duration decreasing as $u^{-1 / 2}$, and pulse peak power and energy, growing as $u^{2}$ and $u^{3 / 2}$ respectively [24,25,39]. 


\section{PULSE PROPAGATION VELOCITY}

The pulse propagation velocity is given by the slope $v=$ $d \zeta / d u$ of the field envelope in the plane $(\zeta, u)$. According to the definition, a point moving with velocity $v$ in the space $u, \zeta=\zeta_{0}+u v$, has a velocity

$$
v_{L}=\frac{d z}{d t}=\frac{d}{d t}\left(\zeta l_{2}+u l_{1}\right) \simeq c\left[1+\frac{\lambda_{0}}{\lambda_{u}}(v-1)\right]
$$

in the coordinates $z, t$. The condition $v_{L}=c$ is therefore equivalent to $v \simeq 1$. The change of slope at $u \approx 2$, visible in Fig. 1(a), corresponds to a threshold where the amplified field becomes larger then the initial seed and the observed propagation velocity reduces from $v \simeq 1$ to $v=v_{e} \simeq 1 / 3$, as predicted by the theory [22]. The velocity changes again at saturation, where the pulse splits. Saturation occurs where the bunching is locally phase shifted by $\sim \pi / 2$ with respect to the field. At $u=u_{s}$, where the peak of the pulse at $\zeta=\zeta_{s}$ is saturated, the wings of the pulse $\left(\zeta>\zeta_{s}\right.$ and $\left.\zeta<\zeta_{s}\right)$ where $|a(\zeta, u)|<\left|a_{s}\right|$ are still growing in exponential regime. For $u>u_{s}$, the peaks of the two modes delimit a saturated central region and their position is determined by the condition $|a(\zeta, u)|=a_{s}$, where $a_{s}$ is the peak field at saturation. We have calculated the peak position of the leading and trailing modes, by approximating the field envelope at saturation with a Gaussian distribution still growing exponentially with $u$, and drifting in $\zeta$ with velocity $v$, i.e.,

$$
|a(\zeta, u)|=a_{s} e^{\left[-\left(\zeta-\zeta_{s}-v\left(u-u_{s}\right)\right)^{2} / 4 \sigma_{\zeta s}^{2}+\sqrt{3}\left(u-u_{s}\right) / 2\right]} .
$$

The roots of $|a(\zeta, u)|=a_{s}$ are

$$
\zeta_{ \pm}(u)=\zeta_{s}+\left(u-u_{s}\right) v \pm \sigma_{\zeta s}\left[2 \sqrt{3}\left(u-u_{s}\right)\right]^{1 / 2},
$$

and are shown as a white line in Fig. 1 . When $\sigma_{\zeta 0}>1$, the amplification effect on the pulse profile before saturation is modest. After saturation only the pulse region between the two roots in Eq. (4) undergoes into a deep change. The front and rear wings, outside the saturated region, still preserve their original Gaussian shape and the function $\zeta_{ \pm}(u)$, with $v=v_{e}$, reasonably matches the positions of the split peaks [Fig. 1(b), white line]. Conversely, for $\sigma_{\zeta 0}<1$ at saturation [Fig. 1(a), white line], the effect of the slippage becomes dominant, the position of the front peak drifts with a velocity larger than $v_{e}$ and the pulse profile is distorted with a reduced rise time, reflecting the pulse shortening characteristic of superradiance. The pulse velocity in exponential gain regime is dominated by the propagation of the density modulated electron beam that amplifies the field. After saturation, the exponential amplification is suppressed, the radiation is actively modulating the beam and is only weakly modified by the interaction; the pulse velocity converges then to the velocity of light. The expression in Eq. (4) may therefore still reproduce the behavior of the front peak, by setting $v=1$ and by multiplying the pulse duration by a scaling factor reflecting the progressive pulse shortening effect, i.e.,

$$
\zeta_{+c}(u)=\zeta_{s}+\left(u-u_{s}\right) \pm \beta \sigma_{\zeta s}\left[2 \sqrt{3}\left(u-u_{s}\right)\right]^{1 / 2}
$$

The value of the coefficient $\beta$ depends on the value of $\sigma_{\zeta 0}$ and varies in the range $(0.25-0.30)$ for $\sigma_{\zeta 0} \lesssim 1$. Equation (5) reproduces the asymptotic behavior of the peak position with $\beta=0.25$ [Fig. 1(a), white dashed line], but also provides a peak propagation velocity $\frac{d}{d u} \zeta_{+c}(u)>1$, which converges to one only in the limit $u \rightarrow \infty$.

In Fig. 2 we have plotted the velocity of the peak from the solution of Eq. (1) (red line), the velocity of the peak calculated from Eq. (4) (black line) and from Eq. (5) (black dashed line). After saturation, the velocity from the corrected Eq. (5) matches the theoretical peak velocity. The fact that the peak moves faster than the light in vacuum may be interpreted as the result of a change in shape of the pulse during evolution. In Fig. 2 we have also plotted the velocity of the energy "center of mass," also indicated as "centrovelocity" [40] for the entire pulse (blue line), and for the peak region only (green line). The latter is defined as the interval with an extension of two full-width at halfmaximum (f.w.h.m.) centred at the position of the peak. In both cases, we have an apparent propagation of energy at "superluminal" velocities over distances of several lengths $l_{1}$. This may appear as a violation of the principle of relativity or of causality, but the energy is not effectively drifting over the electrons at "superluminal" speed, as the energy is uniformly stored as electron kinetick energy and what we observe is the conversion of electron kinetic

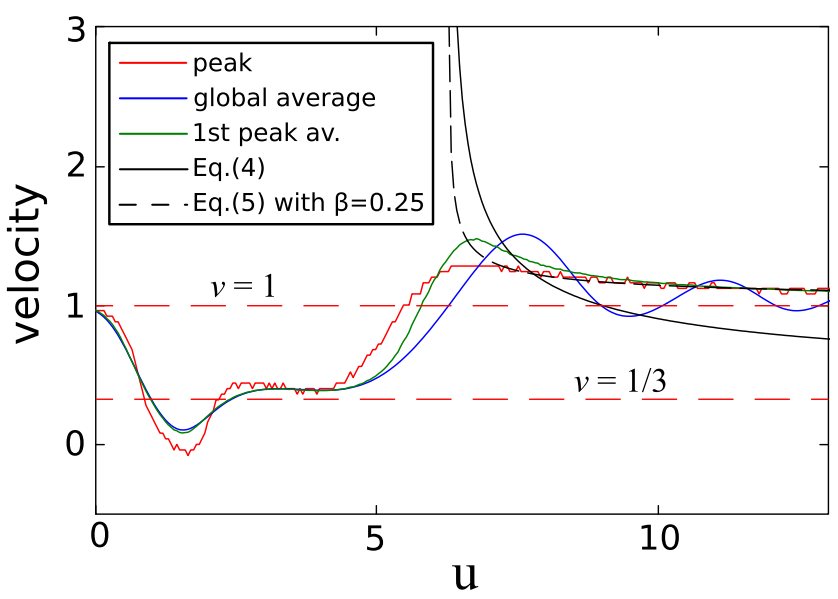

FIG. 2. Pulse velocity calculated from the system of Eq. (1) for the peak of the pulse (red line), for the energy barycenter of the entire pulse (blue line) and the barycenter of the main peak only (green line). The black continuous/dashed lines represent the pulse peak velocity $\frac{d}{d u} \zeta_{+c}(u)$ calculated from Eqs. (4) and (5) respectively. The horizontal red dashed lines indicate the velocity of light in vacuum $(v=1)$ and the exponential gain regime theoretical velocity $(v=1 / 3)$. 
energy into electromagnetic energy of the wave, combined with a change of shape of the electromagnetic energy distribution. The superluminarity is not violating any causality condition; it corresponds to a pulse shape distortion in an active medium [41], where the leading edge propagates at $v=1$ and is actively modulating and dragging energy from the beam current, the trailing edge is instead out of phase with the induced modulation and decreases by returning part of its energy to the beam.

The pulse average velocity, blue line in Fig. 2, shows periodic oscillations as a function of the coordinate $u$, suggesting a non trivial behavior of the beam diffraction index. In order to understand the properties of the saturated electron beam dispersive medium, we calculate the pulse group velocity from the phase evolution of the field, i.e.,

$$
v_{g}=\frac{d \omega}{d \kappa}=\frac{d}{d \kappa}\left[\frac{d}{d u} \arg (\tilde{a})\right],
$$

where $\tilde{a}(\kappa, u)=\int_{-\infty}^{+\infty} \exp (i \kappa \zeta) a(\zeta, u) d \zeta$ is the Fourier transform of $a(\zeta, u)$.

In Fig. 3 the power spectrum $|\tilde{a}(\kappa, u)|^{2}$ and the group velocity, versus the normalized wave-vector $\kappa=\left(k-k_{0}\right) /$ $2 \rho_{\text {fel }} k_{0}$, are shown for $\sigma_{\zeta 0}=0.82$ [as in Fig. 1(a)]. During the exponential growth, the gain process filters the spectrum which narrows up to $u \approx 8$. Here we have the formation of a broad background and a narrower redshifted structure. The white dashed lines in Fig. 3(b) delimit the region where most of the pulse energy flows. In agreement with the theory, we have $v_{g} \sim 1 / 3$ in exponential regime. At saturation, we observe a modulation of the group velocity as a function of the frequency, i.e., a modulation of the group velocity refractive index
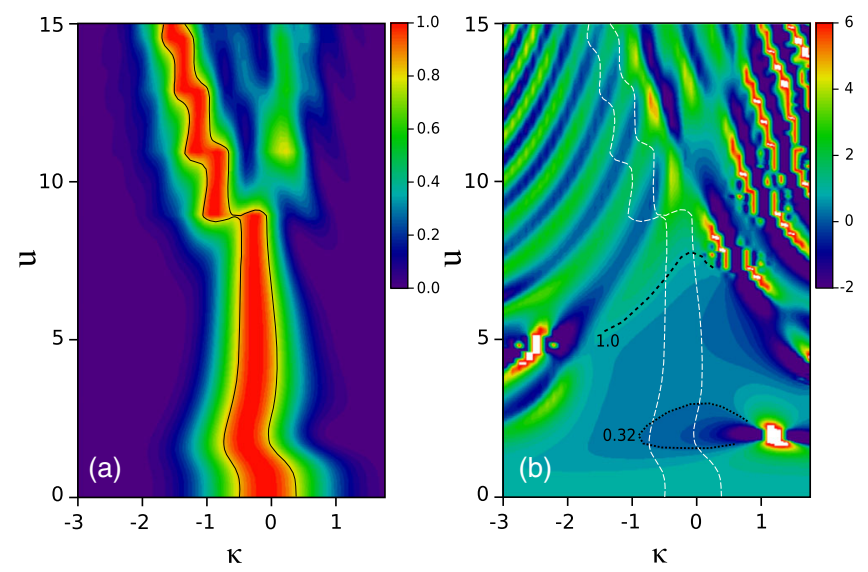

FIG. 3. Power spectrum (a) and group velocity (b) corresponding to the case of Fig. 1(a) vs $u, \kappa$. The power $P$ is normalized at each position in $u$. The white dashed line in (b) represents the power contour line, in black in (a), corresponding to the level $\simeq 0.8$. The black dotted and dashed contour lines in (b) delimit the transitions to exponential regime $\left(v_{g} \sim 1 / 3\right)$ and to postsaturation (at $u \sim 7-8$ ) where $v_{g}>1$. $n_{g}=1 / v_{g}$, suggesting the existence of spectral regions of anomalous dispersion. The group velocity $v_{g}$ increases and becomes larger than one at the splitting position $(u=7$, Fig. 1). The radiation energy flows from spectral regions where $v_{g}>1$ to regions where $v_{g}<1$, corresponding to the discrete redshifts in the evolution of the spectrum. The condition for preserving the causality principle is that the "signal" velocity [40], i.e., the "information" velocity, do not exceed the velocity of light in vacuum and in superradiance the "front" side of the pulse propagates at $c$. The consequence of this is the spectral broadening occurring after saturation, which reflects a progressive narrowing of the pulse coherence length. This contributes to clarify the questions raised in $[39,42]$ justifying the validity of the scaling relations of superradiance in FELs expressed in terms of the coordinate $u$ given in [24], i.e., peak power, pulse energy and duration proportional to $u^{2}, u^{3 / 2}$, and $u^{-1 / 2}$ respectively.

In single pass FELs superluminal propagation occurs over few gain lengths and the longitudinal offset per gain length is of the order of the cooperation length. It is therefore not trivial to find an experimental evidence of this "superluminar" behavior. The situation is different in FEL oscillators where the optical cavity stacks optical pulses accumulating the offset over a large number of roundtrips. In FEL oscillators, the extraction efficiency depends on the cavity detuning $\delta L$ from the perfect synchronism. This perfect synchronism corresponds to the condition where the cavity round-trip time matches the separation between electron bunches, which is given by a multiple of the period of the radio frequency. The maximum power extracted from a FEL oscillator in small gain regime, is expected at $\delta L<0$ [30]. The small negative cavity detuning $\delta L$ compensates for the delay induced by the gain refractive index. Super-radiance effects in high gain oscillators were predicted and observed at the upper extreme of the cavity tuning range, i.e., at $\delta L \sim 0$ [43-45]. In the FEL oscillator experiment [46], together with the FEL output power, the cavity length was accurately determined with interferometric methods. The experiment has shown that the FEL maximum efficiency was reached with the cavity detuning $\delta L=0$, and that the FEL was delivering power even at $\delta L>0$. Despite the observed peak in efficiency, the lasing dynamics at $\delta L \sim 0$ was affected by instability, as a consequence of noise injection which we may associate to the causality condition.

\section{PULSE STRUCTURE AND TAIL SUPPRESSION}

We analyze the process in detail by inspecting the pulse structure in Fig. 4, where we have a snapshot of the field of Fig. 1(a) at $u=15$. The field envelope (black line) assumes the shape of a self-similar solitary wave with a main peak in the leading edge (right side), followed by a sequence of secondary peaks. The number of these peaks depends on 
the saturation depth. Approximated expressions for the main peak were calculated in Refs. [32,33] but these models were not suited to follow the nonlinear dynamics in the tail. The structure of the tail results from an interferential process where the relative phases of field and bunching lead to alternated emission and absorption processes. We may distinguish a total of six peaks in Fig. 4; the first three on the right side correspond to front side modes, and the three in the tail correspond to trailing modes that are not visible in Fig. 1(a) where the field intensity (and not the amplitude) is shown. The bunching factor (dashedorange) is high $(|b| \approx 0.5)$ in the entire pulse region. The amplitude asymmetry between the two sets of modes depends on the large slippage parameter, favoring the front side slipping on fresh electrons. On the broad range of the entire pulse, the phase (light green line) shows a quadratic behavior. On the leading edge, peaks (b), (d) the phase has a linear trend with a phase shift of $\approx \pi$ occurring at the transitions between the subpulses, positions (c) and (e) (green dots and dark-green enhanced line). This is interpreted as the fact that the bunching induced by the main peak grows up to a maximum between (a) and (b) and after (b) drifts out of phase with the existing field. The field therefore decreases until it changes sign in (c). At this position in $\zeta$, the bunched electrons have a phase mismatch of $\pi$ with the field of the main peak [Fig. 4(c)] and produce a second peak with a $\pi$ shift in phase. The same process is repeated in a new cycle between (d) and (e), but at every cycle the trapped fraction of the charge decreases because of the induced energy spread, and the amplitudes of the peaks decrease accordingly. These secondary pulses form a pedestal that has an extension approximately given by the distance between the two roots in Eq. (4), $\Delta \sim \zeta_{+c}-\zeta_{-}$. This distance can be significantly longer than the duration of the leading pulse and can carry a large fraction of the total pulse energy. In Fig. 5 we have plotted the duration of the pulse represented in Fig. 1(a) in three different ways. Line A represents the r.m.s. pulse duration from the solution of Eq. (1). Line B (red) represents the r.m.s. duration associated to a Gaussian with the same f.w.h.m. of the pulse power distribution, i.e., it is width is not sensitive to the pulse tail. The two functions are reasonably in agreement during the exponential growth, but they diverge after saturation, where the tail builds up. It is worth to observe that the duration of a Fourier transform limited Gaussian with the r.m.s. spectral width calculated from the pulse spectral distribution of Fig. 3, line C (black), closely reproduces the behavior of line $\mathrm{B}$ even after saturation. For the same reason, the time-bandwidth product (TBP) of the pulse, plotted in Fig. 5(b) from the r.m.s. duration and spectral width (blue), and from the r.m.s. duration derived from the f.w.h.m and the r.m.s. spectral width (red), show a completely different behavior after saturation. The first grows for the increase of the tail energy content; the second remains close to the theoretical minimum $T B P$ of an ideal Gaussian power distribution $(=0.5)$. Experimentally, in the limits of the "flat beam" assumption used in the integration of Eq. (1), the spectral width can be used to infer the
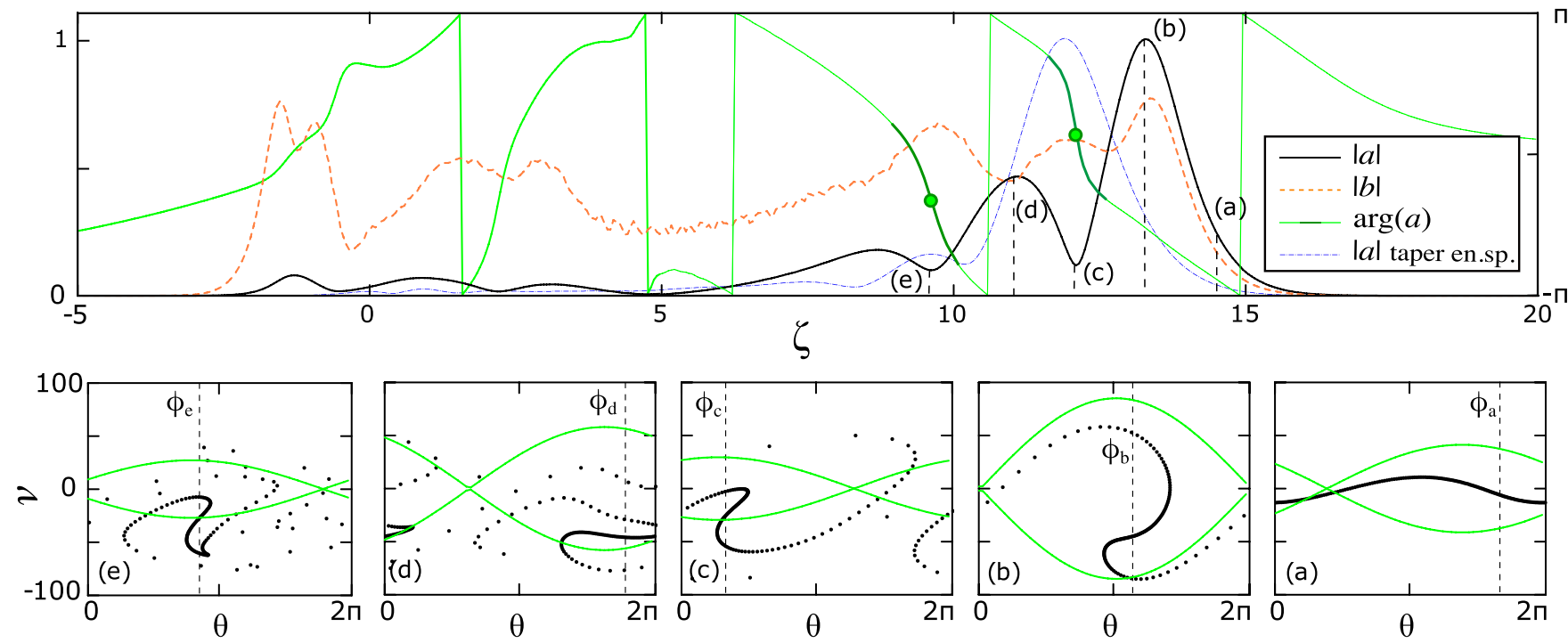

FIG. 4. Field amplitude normalized to the peak (black line), field phase (green-right scale) and modulus of the bunching factor (dashedorange) vs $\zeta$, are shown (at $u=15$ ). The field phase at the positions (c) and (e) corresponding to the field amplitude minima in the front mode, is indicated by the green dot and the phase line is in evidence in dark green in the regions of phase shifts between the peaks. The e-beam phase spaces at the positions (a-e) are plotted in the bottom row figures. The green lines in (a-e) represent the separatrices $S_{ \pm}=$ $\pm \sqrt{2[\operatorname{Re}(a) \sin (\theta)+\operatorname{Im}(a) \cos (\theta)+|a|]}$ showing the field phase and the fraction of trapped electrons. The phase of the bunching factor $b$ at each position is indicated by $\phi_{a, b, c, d, e}=(1.65,1.15,0.33,1.78,0.85)$ respectively (vertical black-dashed lines). The blue-dashed line represents the field envelope in presence of tapered energy spread, according to the rule given in Eq. (7), with $\alpha_{t}=4 \%$. 

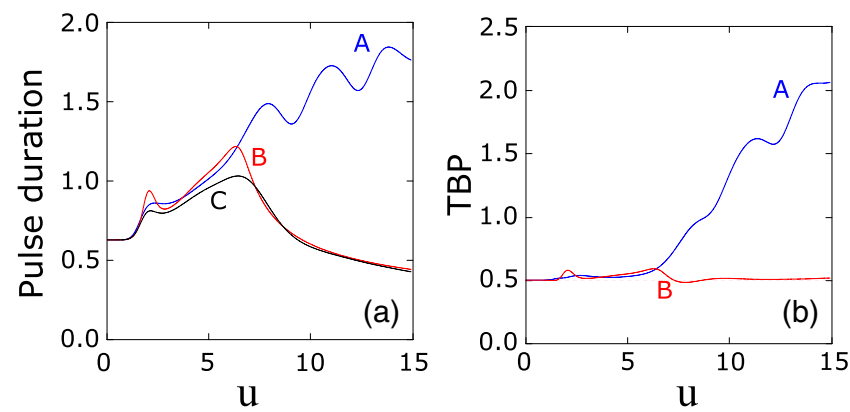

FIG. 5. (a) Duration of the pulse represented in Fig. 1(a); line A (blue) represents the r.m.s. pulse duration from the integration of Eq. (1); line B (red) represents the r.m.s. duration of a Gaussian distribution (in power) with the same f.w.h.m. of the pulse; line C (black), represents the duration of a Fourier transform limited Gaussian with the r.m.s. spectral width calculated from the spectral distribution in Fig. 3. (b) Time-bandwidth product (TBP) calculated from the r.m.s. duration and spectral width (A-blue) and from the scaled f.w.h.m duration and the r.m.s. spectral width (B-red), are completely different after saturation.

duration of the main peak; this fact has some relevance, as the measurement of the radiation spectrum is generally much simpler than the direct measurement of the pulse duration [47]. The entire pulse however, is far from the Fourier limit, and the reason is the existence of the long tail which would be a source of background and noise in a time resolved experiment.

In the example of Fig. 4, black line, we have calculated the radiation energy of the tail $E_{\text {tail }}$ and the ratio $w_{\Delta}=E_{\text {tail }} / E_{\text {tot }} \approx 23 \%$, by defining the tail region as the interval from $-\infty$ to the first minimum in (c). A Gaussian fit of the main peak has an r.m.s. duration of $0.44 l_{c}$ but the effective r.m.s. pulse duration, including the tail, is $1.76 l_{c}$ and the r.m.s. TBP is 2.06 (see Fig. 5). Energy chirp or undulator taper have a small effect on the energy content of the tail. The reason is that a change in resonance modifies the phase matching condition, varying the temporal structure of the sub-pulses, but not their average amplitudes. On the other hand, these subpulses are affected by the inhomogeneous broadening associated to beam energy spread. In Fig. 6 we have plotted the $T B P$, the fractional energy content of the tail $w_{\Delta}$ and the relative reduction of the energy in the main peak, i.e., $\eta=E_{\text {peak }}\left(\sigma_{\epsilon}\right) / E_{\text {peak }}(0)$ as a function of the beam energy spread.

An induced energy spread $\sigma_{e}$ causes a reduction of the pulse energy and delays the onset of saturation, but it is also effective in reducing $w_{\Delta}$. A good compromise is found at $\sigma_{e} \simeq \rho_{\text {fel }} / 2$ where $w_{\Delta} \approx 2.5 \%$ and the pulse energy in the main peak decreases by $1-\eta \sim 22 \%$. A modest energy spread is not beneficial during exponential growth but only in the post saturation dynamics. We explored a condition where the energy spread on the beam is not uniform, but increases locally where the pulse drifts into saturation. This may be realized by, e.g., tapering the energy spread with a

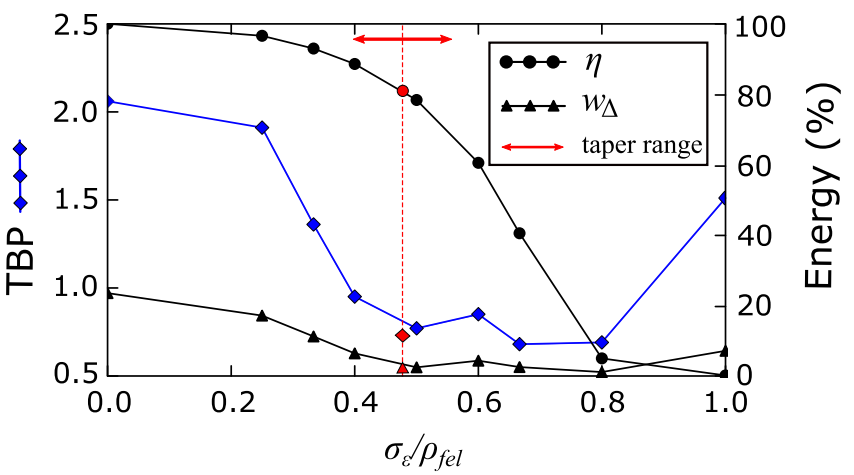

FIG. 6. Effect of the beam energy spread $\sigma_{\epsilon}$ on the relative energy in the main peak $E_{\text {loss }}$ (black circles), on the fractional tail energy $w_{\Delta}$ (black triangles) and on the pulse r.m.s. time bandwidth product (blue squares). The red symbols represent the same parameters in presence of tapered energy spread according to the rule given in Eq. (7), with $\alpha_{t}=4 \%$. The red dashed line is centered on the range spanned by the local value of the energy spread during the pulse evolution. This range is indicated by the horizontal red-arrow.

laser heater [48], or by triggering the pulse growth at a specific position along the beam where it naturally slips into a larger energy spread region. We assumed a linear growth of the slice energy spread starting at saturation $\zeta=\zeta_{s}$, according to the following expression,

$$
\sigma_{\epsilon}=2 \rho_{\mathrm{fel}} / 5\left[1+\alpha_{t} \Theta\left(\zeta-\zeta_{s}\right)\left(\zeta-\zeta_{s}\right)\right]
$$

We explored the behavior of the system vs the parameter $\alpha_{t}$ and the case corresponding to $\alpha_{t}=4 \%$ is close to the optimum. It is indicated in Fig. 6 by the red dashed line, centered on the range spanned by the local value of the energy spread during the pulse evolution (red-arrow). In this case the tail suppression is larger, $w_{\Delta} \simeq 2.1 \%$ and the r.m.s. pulse duration is reduced to $0.8 l_{c}$, with a $T B P=0.73$. The energy loss in the main peak is $1-\eta \sim$ $19 \%$ with respect to the energy spread free case. A modest energy reduction could be compensated by an increase of the length of the post-saturation propagation distance. The associated field envelope is represented in Fig. 4 by the blue-dashed line.

We have summarized the values of the parameters $\eta, w_{\Delta}$ and $T B P$ in the three conditions: no energy spread/ optimum energy spread/tapered energy spread in Table I.

TABLE I. Parameters $\eta, w_{\Delta}$ and TBP in the three conditions: no energy spread ( $n$ ull $)$, optimum energy spread $\left(\rho_{\text {fel }} / 2\right)$, and tapered energy spread according to Eq. (7) (taper), with $\alpha_{t}=4 \%$.

\begin{tabular}{lccc}
\hline \hline Energy spread & Null & $\rho_{\text {fel }} / 2$ & Taper \\
\hline$\eta$ & $100 \%$ & $79 \%$ & $81 \%$ \\
$w_{\Delta}$ & $23 \%$ & $2.5 \%$ & $2.1 \%$ \\
$T B P$ & 2.06 & 0.77 & 0.73 \\
\hline \hline
\end{tabular}




\section{CONCLUSIONS}

In conclusion, the superradiant spike dynamics was investigated finding conditions where the group velocity and the centrovelocity of the light become larger than $c$. The pulse structure and the structure of the tail was analyzed showing that a controlled energy spread could reduce the tail energy. We explored an ideal case, where the beam current and the beam energy longitudinal distributions are uniform. The pulse shape should be affected by a beam energy chirp or by a tapered undulator. A tailored taper could retard the phase mismatch occurring along the pulse and may be used to fine tune both the pulse energy and its duration. Advanced undulator taper techniques [49] have shown the possibility to extract a large fraction of the beam energy in a seeded FEL amplifier and should be investigated to control the post saturation dynamics of a single spike, one of the keys to extend the FEL performances to the level required by some of the most challenging applications.

[1] P. Emma, R. Akre, J. Arthur, R. Bionta, C. Bostedt, J. Bozek, A. Brachmann, P. Bucksbaum, R. Coffee, F.-J. Decker et al., First lasing and operation of an ångstromwavelength free-electron laser, Nat. Photonics 4, 641 (2010).

[2] T. Ishikawa, H. Aoyagi, T. Asaka, Y. Asano, N. Azumi, T. Bizen, H. Ego, K. Fukami, T. Fukui, Y. Furukawa et al., A compact X-ray free-electron laser emitting in the subångström region, Nat. Photonics 6, 540 (2012).

[3] M. L. Grünbein, J. Bielecki, A. Gorel, M. Stricker, R. Bean, M. Cammarata, K. Dörner, L. Fröhlich, E. Hartmann et al., Megahertz data collection from protein microcrystals at an X-ray free-electron laser, Nat. Commun. 9, 3487 (2018).

[4] W. Ackermann et al., Operation of a free-electron laser from the extreme ultraviolet to the water window, Nat. Photonics 1, 336 (2007).

[5] E. Allaria et al., Highly coherent and stable pulses from the FERMI seeded free-electron laser in the extreme ultraviolet, Nat. Photonics 6, 699 (2012).

[6] E. Allaria et al., Two-stage seeded soft-X-ray free-electron laser, Nat. Photonics 7, 913 (2013).

[7] R. Bonifacio, C. Pellegrini, and L. M. Narducci, Collective instabilities and high-gain regime in a free electron laser, Opt. Commun. 50, 373 (1984).

[8] M. Xie, in Proceedings of the Particle Accelerator Conference, Dallas, TX, 1995 (IEEE, New York, 1995), Vol. 1, pp. 183-185.

[9] R. Bonifacio, L. De Salvo, P. Pierini, N. Piovella, and C. Pellegrini, Spectrum, Temporal Structure, and Fluctuations in a High-Gain Free-Electron Laser Starting from Noise, Phys. Rev. Lett. 73, 70 (1994).

[10] H. J. Wörner, C. A. Arrell, N. Banerji, A. Cannizzo, M. Chergui, A. K. Das, P. Hamm, U. Keller, P. Kraus et al., Charge migration and charge transfer in molecular systems, Structural dynamics (Melville, N.Y.) 4, 061508 (2017).
[11] R. Neutze, R. Wouts, D. van der Spoel, E. Weckert, and J. Hajdu, Potential for biomolecular imaging with femtosecond X-ray pulses, Nature (London) 406, 752 (2000).

[12] A. Fratalocchi and G. Ruocco, Single-Molecule Imaging with X-Ray Free-Electron Lasers: Dream or Reality?, Phys. Rev. Lett. 106, 105504 (2011).

[13] H. N. Chapman, P. Fromme, A. Barty, T. A. White, R. A. Kirian, A. Aquila, M. S. Hunter, J. Schulz, D. P. DePonte, $\mathrm{U}$. Weierstall et al., Femtosecond X-ray protein nanocrystallography, Nature (London) 470, 73 (2011).

[14] P. Emma, K. Bane, M. Cornacchia, Z. Huang, H. Schlarb, G. Stupakov, and D. Walz, Femtosecond and Subfemtosecond X-Ray Pulses from a Self-Amplified SpontaneousEmission-Based Free-Electron Laser, Phys. Rev. Lett. 92, 074801 (2004).

[15] A. A. Zholents and W. M. Fawley, Proposal for Intense Attosecond Radiation from an X-Ray Free-Electron Laser, Phys. Rev. Lett. 92, 224801 (2004).

[16] A. A. Zholents and G. Penn, Obtaining attosecond x-ray pulses using a self-amplified spontaneous emission free electron laser, Phys. Rev. Accel. Beams 8, 050704 (2005).

[17] E. L. Saldin, E. A. Schneidmiller, and M. V. Yurkov, Selfamplified spontaneous emission FEL with energy-chirped electron beam and its application for generation of attosecond x-ray pulses, Phys. Rev. Accel. Beams 9, 050702 (2006).

[18] J. B. Rosenzweig, D. Alesini, G. Andonian, M. Boscolo, M. Dunning, L. Faillace, M. Ferrario, A. Fukusawa, L. Giannessi, E. Hemsing et al., Generation of ultra-short, high brightness electron beams for single-spike SASE FEL operation, Nucl. Instrum. Methods Phys. Res., Sect. A 593, 39 (2008).

[19] A. A. Zholents and M. S. Zolotorev, Attosecond x-ray pulses produced by ultra short transverse slicing via laser electron beam interaction, New J. Phys. 10, 025005 (2008).

[20] S. Huang, Y. Ding, Y. Feng, E. Hemsing, Z. Huang, J. Krzywinski, A. A. Lutman, A. Marinelli, T. J. Maxwell, and D. Zhu, Generating Single-Spike Hard X-Ray Pulses with Nonlinear Bunch Compression in Free-Electron Lasers, Phys. Rev. Lett. 119, 154801 (2017).

[21] A. A. Lutman, M. W. Guetg, T. J. Maxwell, J. P. MacArthur, Y. Ding, C. Emma, J. Krzywinski, A. Marinelli, and Z. Huang, High-Power Femtosecond Soft X Rays from FreshSlice Multistage Free-Electron Lasers, Phys. Rev. Lett. 120, 264801 (2018).

[22] R. Bonifacio, L. De Salvo Souza, P. Pierini, and N. Piovella, The superradiant regime of a FEL: Analytical and numerical results, Nucl. Instrum. Methods Phys. Res., Sect. A 296, 358 (1990).

[23] R. Bonifacio, F. Casagrande, G. Cerchioni, L. De Salvo Souza, P. Pierini, and N. Piovella, Physics of the high-gain FEL and superradiance, Riv. Nuovo Cimento Soc. Ital. Fis. 13, 1 (1990).

[24] L. Giannessi, P. Musumeci, and S. Spampinati, Nonlinear pulse evolution in seeded free-electron laser amplifiers and in free-electron laser cascades, J. Appl. Phys. 98, 043110 (2005).

[25] T. Watanabe, X. J. Wang, J. B. Murphy, J. Rose, Y. Shen, T. Tsang, L. Giannessi, P. Musumeci, and S. Reiche, 
Experimental Characterization of Superradiance in a Single-Pass High-Gain Laser-Seeded Free-Electron Laser Amplifier, Phys. Rev. Lett. 98, 034802 (2007).

[26] L. Giannessi, M. Bellaveglia, E. Chiadroni, A. Cianchi, M. E. Couprie, M. Del Franco, G. Di Pirro, M. Ferrario, G. Gatti, M. Labat et al., Superradiant Cascade in a Seeded Free-Electron Laser, Phys. Rev. Lett. 110, 044801 (2013).

[27] C. G. B. Garrett and D. E. McCumber, Propagation of a Gaussian light pulse through an anomalous dispersion medium, Phys. Rev. A 1, 305 (1970).

[28] F. T. Arecchi and R. Bonifacio, Theory of optical maser amplifiers, IEEE J. Quantum Electron. 1, 169 (1965).

[29] H. Haus, Noise in free-electron laser amplifier, IEEE J. Quantum Electron. 17, 1427 (1981).

[30] G. Dattoli, A. Marino, A. Renieri, and F. Romanelli, Progress in the Hamiltonian picture of the free-electron laser, IEEE J. Quantum Electron. 17, 1371 (1981).

[31] G. Dattoli, D. Levi, and M. Mattioli, Free electron lasers and soliton propagation, Phys. Lett. A 256, 253 (1999), cited By 2.

[32] J. Wu, J. B. Murphy, X. Wang, and K. Wang, Exponential growth, superradiance, and tunability of a seeded free electron laser, Opt. Express 16, 3255 (2008).

[33] N. Piovella, A hyperbolic secant solution for the superradiance in free electron lasers, Opt. Commun. 83, 92 (1991).

[34] L. Giannessi, in Proceedings of FEL 2006 Conference Germany, http://www.jacow.org (2006), p. 91.

[35] E. L. Saldin, E. A. Schneidmiller, and M. V. Yurkov, Statistical properties of radiation from VUV and X-ray free electron laser, Opt. Commun. 148, 383 (1998).

[36] M. Labat, N. Joly, S. Bielawski, C. Szwaj, C. Bruni, and M. E. Couprie, Pulse Splitting in Short Wavelength Seeded Free Electron Lasers, Phys. Rev. Lett. 103, 264801 (2009).

[37] G. De Ninno, B. Mahieu, E. Allaria, L. Giannessi, and S. Spampinati, Chirped Seeded Free-Electron Lasers: Self-Standing Light Sources for Two-Color Pump-Probe Experiments, Phys. Rev. Lett. 110, 064801 (2013).

[38] B. Mahieu, E. Allaria, D. Castronovo, M. B. Danailov, A. Demidovich, G. De Ninno, S. Di Mitri, W. M. Fawley, E. Ferrari, L. Fröhlich et al., Two-colour generation in a chirped seeded free-electron laser: a close look, Opt. Express 21, 22728 (2013).
[39] T. Watanabe, X. J. Wang, J. B. Murphy, J. Rose, Y. Shen, T. Tsang, L. Giannessi, P. Musumeci, and S. Reiche, Watanabe et al. Reply:, Phys. Rev. Lett. 99, 029502 (2007).

[40] R. L. Smith, The velocities of light, Am. J. Phys. 38, 978 (1970).

[41] R. Y. Chiao, Superluminal (but causal) propagation of wave packets in transparent media with inverted atomic populations, Phys. Rev. A 48, R34 (1993).

[42] R. Bonifacio, F. Casagrande, D. A. Jaroszynski, B. W. J. McNeil, N. Piovella, and G. R. M. Robb, Comment on "Experimental Characterization of Superradiance in a Single-Pass High-Gain Laser-Seeded Free-Electron Laser Amplifier", Phys. Rev. Lett. 99, 029501 (2007).

[43] D. A. Jaroszynski, C. R. Pidgeon, M. F. Kimmitt, G. Spiers, M. W. Poole, and M. G. Kelliher, Measurements of the dispersive and absorptive properties of the freeelectron-laser gain medium, Nucl. Instrum. Methods Phys. Res., Sect. A 296, 62 (1990).

[44] P. L. Ottaviani, S. Pagnutti, G. Dattoli, E. Sabia, V. Petrillo, P. J. M. van der Slot, S. Biedron, and S. Milton, Deep saturated Free Electron Laser oscillators and frozen spikes, Nucl. Instrum. Methods Phys. Res., Sect. A 834, 108 (2016).

[45] G. Dattoli, P. L. Ottaviani, and S. Pagnutti, High gain oscillators: Pulse propagation and saturation, J. Appl. Phys. 101, 103109 (2007).

[46] N. Nishimori, R. Hajima, R. Nagai, and E. J. Minehara, Sustained Saturation in a Free-Electron Laser Oscillator at Perfect Synchronism of an Optical Cavity, Phys. Rev. Lett. 86, 5707 (2001).

[47] P. Finetti, H. Höppner, E. Allaria, C. Callegari, F. Capotondi, P. Cinquegrana, M. Coreno, R. Cucini, M. B. Danailov, A. Demidovich et al., Pulse Duration of Seeded Free-Electron Lasers, Phys. Rev. X 7, 021043 (2017).

[48] A. Marinelli, R. Coffee, S. Vetter, P. Hering, G. N. West, S. Gilevich, A. A. Lutman, S. Li, T. Maxwell, J. Galayda, A. Fry, and Z. Huang, Optical Shaping of X-Ray FreeElectron Lasers, Phys. Rev. Lett. 116, 254801 (2016).

[49] J. Duris, A. Murokh, and P. Musumeci, Tapering enhanced stimulated superradiant amplification, New J. Phys. 17, 063036 (2015). 